\title{
Spatiotemporal Light Control with Frequency-Gradient Metasurfaces
}

Authors: Amr M. Shaltout ${ }^{1}$, Konstantinos G. Lagoudakis ${ }^{2,3}$, Jorik van de Groep ${ }^{1}$, Soo Jin Kim ${ }^{1,4}$, Jelena Vučković ${ }^{2}$, Vladimir M. Shalaev ${ }^{5}$, and Mark L. Brongersma ${ }^{1, *}$

\author{
Affiliations: \\ ${ }^{1}$ Geballe Laboratory for Advanced Materials, Stanford University, Stanford, California 94305, USA \\ ${ }^{2}$ Ginzton Laboratory, Stanford University, Stanford, California 94305, USA \\ ${ }^{3}$ Department of Physics, University of Strathclyde, Glasgow G4 0NG, UK \\ ${ }^{4}$ School of Electrical Engineering, Korea University, Seoul 02841, South Korea \\ 5 School of Electrical \& Computer Engineering and Birck Nanotechnology Center, Purdue University, \\ West Lafayette, IN 47907, USA \\ * Correspondence to: brongersma@stanford.edu
}

\begin{abstract}
:
The capability of on-chip wavefront modulation has the potential to revolutionize many optical device technologies. However, the realization of power-efficient phase-gradient metasurfaces offering full-phase modulation $(0-2 \pi)$ and high operation speeds remains elusive. We present an approach to continuously steer light based on creating a virtual frequency-gradient metasurface by combining a passive metasurface with an advanced frequency-comb source. Spatiotemporal redirection of light naturally occurs as optical phase-fronts reorient at a speed controlled by the frequency-gradient across the virtual metasurface. An experimental realization of laser beam steering with a continuously changing steering-angle is demonstrated with a single metasurface over an angle of $25^{\circ}$ in just 8 picoseconds. This work can support integrated-on-chip solutions for spatiotemporal optical control, directly impacting vital applications including LIDAR, autonomous vehicles, and Augmented/Virtual systems.
\end{abstract}




\section{One Sentence Summary:}

Frequency-gradient metasurfaces are used to realize ultrafast and continuous laser beam steering.

\section{Main Text:}

Phase-gradient metasurfaces (1) have demonstrated the ability to shape optical wavefronts in light bending devices (2), meta-lenses (3-5), meta-holograms (6), wave-plates (7-9), as well as devices with chiral (10-12) responses. These functions are achieved by controlling the scattering of light waves from a dense array of nanoscale optical antennas that make up a metasurface. Most metasurfaces to date are passive and their functions are fixed during their fabrication. Many optical applications require dynamic manipulation of light waves and an expansion to spatiotemporallyvarying metasurfaces could open many new opportunities for the metamaterials field (13-21). However, the development of dynamic metasurfaces has been hampered significantly by the challenge of realizing antenna structures capable of providing large changes in the amplitude and phase $(0-2 \pi)$ of scattered light with an external stimulus. In addition, there is a major technological challenge in addressing individual nanoscale antenna within a large-area metasurface device in terms of the electrical wiring and power consumption. Our approach towards spatiotemporal optical control completely avoids the challenges with actively controlling a metasurface.

We start by expanding on the basic concepts underlying the operation of existing phasegradient metasurfaces and illustrate how spatiotemporal optical patterns can be manipulated by controlling the interference of waves in four-dimensional (4D) space-time. We first replace the typical continuous wave $(\mathrm{CW})$ source used to illuminate a metasurface with a frequency-comb source. Such a source features a set of equally-spaced and phase-locked frequency lines. A passive metasurface is engineered to interact with such a frequency-comb source to fashion distinct spatial 
field profiles for each of the spectral lines in the comb. Because the spectral lines are phase-locked, their individual spatial patterns constructively interfere to generate a 4D optical pattern in which the spatial light intensity distribution naturally evolves in time.

Such spatiotemporal metasurfaces add a valuable new dimension to phase-gradient metasurfaces $(22,23)$, illustrated by comparing the simplest implementations of these two optical elements. Figure 1A shows a basic phase-gradient metasurface that imparts a linearly-varying phase-shift on an incident plane wave to redirect it into a desired angle $(2,24)$. Figure 1B shows the corresponding case of a frequency-gradient metasurface constructed from phase-locked optical sources whose frequency is linearly-graded across the metasurface. Their radiation constructively interferes to naturally direct light towards different angles as time progresses.

The mathematical formulation for the operation of spatiotemporal metasurfaces combines the descriptions for spatial mode transformation by a passive metasurface and temporal pulse shaping known from the ultrafast laser physics (25-27). This behavior is conveniently handled in a Fourier synthesis description. Figure 1C demonstrates how a pulse comprised of a set of $2 N+1$ frequency lines $\left(a(t)=\sum_{n=-N}^{n=N} a_{n} e^{-i \omega_{n} t} ; \omega_{n}=\omega_{o}+n \Delta \omega\right) \quad$ interacts with a passive, nanostructured metasurface. The frequencies are centered around an operation frequency $\omega_{o}$ and together they form a pulse $a(t)=A(t) e^{-i \omega_{o} t}$ for which $A(t)=\sum_{n=-N}^{n=N} a_{n} e^{-i n \Delta \omega t}$ is the envelope. In our analysis, a uniform spatial dependence for the pulse amplitude is assumed. Upon illumination, the passive metasurface maps each spectral component into a set of distinct spatial modes $\left(a_{n} \rightarrow b_{n}(\mathbf{r})\right)$, generating a spatiotemporal optical pattern $\left(b(\mathbf{r}, \mathrm{t})=\sum_{n=-N}^{n=N} b_{n}(\mathbf{r}) e^{-i \omega_{n} t}\right)$.

To achieve continous steering of light, we aim to synthesize a new, virtual frequencygradient metasurfaces with a spatial profile $b_{n}(\mathbf{r})$ that mimics an array of optical sources whose frequencies are linearly graded across the surface. The spatiotemporal interference of these sources 
will naturally produce a scanning beam. This can be seen by analyzing the radiation from an array of line sources that are equally spaced at a distance $d$ along the y-axis. The optical source located at the position $\mathbf{r}_{\boldsymbol{n}}=(0, n d)$ in the array radiates at an angular frequency $\omega_{n}$ and a wavenumber $k_{n}=\omega_{n} / c=k_{o}+n \Delta k$, and its optical field distribution is decribed by:

$$
b_{n}(\mathbf{r})=a_{n} G\left(\mathbf{r}-\mathbf{r}_{n}\right) e^{i k_{n}\left(\left|\mathbf{r}-\mathbf{r}_{n}\right|\right)} .
$$

The quantity $a_{n}$ describes the strength of the source, which is proportional to the amplitude of the corresponding frequency line in the incident pulse. The term $G\left(\mathbf{r}-\mathbf{r}_{\boldsymbol{n}}\right)$ is a slowly varying envelope that governs the angular divergence of the radiation pattern and $e^{i k_{n}\left(\left|\mathbf{r}-\mathbf{r}_{n}\right|\right)}$ is a fast varying phase term. With $2 N+1$ sources distributed from $y=-N d$ to $y=N d$, the resulting spatiotemporal pattern in the far-field takes the following form:

$$
b(\mathbf{r}, t)=\sum_{n=-N}^{n=N} b_{n}(\mathbf{r}) e^{-i \omega_{n} t}=\sum_{n=-N}^{n=N} a_{n} G\left(\mathbf{r}-\mathbf{r}_{n}\right) e^{i\left(k_{n}\left|\mathbf{r}-\mathbf{r}_{\boldsymbol{n}}\right|-\omega_{n} t\right)},
$$

where the spatial coordinate $\mathbf{r}=(x, y)=(r \cos \theta,-r \sin \theta)$ is represented in terms of the distance $r$ from the center of the array $(0,0)$, and the clockwise angle $\theta$ (Fig. 1C). As the fields produced by each source evolve in time at different frequencies, the resulting interference pattern will also evolve in time. In the far-field $(r \gg N d)$, the interference pattern can be approximated as (See supplementary text $\mathrm{S} 1)$ :

$$
b(\mathbf{r}, t) \approx A\left(t-\frac{k_{o} d}{\Delta \omega} \sin \theta-\frac{r}{c}\right) G(\mathbf{r}) e^{i\left(k_{o} r-\omega_{o} t\right)} .
$$

The term $A\left(t-\frac{k_{o} d}{\Delta \omega} \sin \theta-\frac{r}{c}\right)$ captures the dynamic beam steering action and shows how the light is directed towards differnent angles $\theta$ at different times $t$. The term $r / c$ accounts for the propagation delay between the frequency-gradient metasurface and the point of detection at a distance $r$. For a cylindrical surface at a fixed distance $r$, the time-depdent steering angle can be in the form: 


$$
\sin \theta=\left(\frac{\Delta \omega}{k_{o} d}\right) t
$$

after translating the time reference through replacing $t-\frac{r}{c}$ with $t$. It follows that the speed of angular steering is directly proportional to the frequency gradient $(\Delta \omega / d)$ across the surface. Eqs $(2,3)$ also demonstrate that the angular beam width is related to the pulse width by the proportionality factor $\left(\frac{\Delta \omega}{k_{o} d}\right)$. For the simple case of cylindrical sources with an omni-directional response $\left(G(\mathbf{r}) \sim \frac{1}{\sqrt{r}}\right)$, and equal amplitudes $\left(a_{n}=1\right)$, we find:

$$
b(\mathbf{r}, t)=\sum_{n=-N}^{N} \frac{e^{i\left(k_{n}\left|r-r_{n}\right|-\omega_{n} t\right)}}{\sqrt{\left|r-r_{n}\right|}}
$$

Figure 1D demonstrates the calculated intensity $I(\mathbf{r}, t)=|b(\mathbf{r}, t)|^{2}$ at different times for an array of 41 cylindrical sources $(2 N+1=41 ; N=20)$, a separation between sources $d=360 \mathrm{~nm}$, a center wavelength of $\lambda_{o}=720 \mathrm{~nm}\left(\left(\right.\right.$ i.e. $\left.\omega_{o}=2 \pi \times 416.66 \mathrm{THz}\right)$, and $\Delta \omega=2 \pi \times 100 \mathrm{GHz}$. When the scanning time is fast enough compared to propagation time of light across the obersveration areas, the light beam appears to be curved. This can be thought of in analogy to the apparent bending of a waterstream emerging from a rapidly rotating water hose. The simulations are discussed in more detail in supplementary text S2, where it is also highlighted that the scanning rate is proportional to the frequency-gradient $(\Delta \omega / d)$.

Figure 2A schematically shows the setup that is used to experimentally demonstrate beam steering. A frequency-gradient virtual metasurface is implemented using a mode locked laser with a frequency-comb spectrum, and a dielectric, phase-gradient metasurface that focuses different spectral components to produce a series of line-sources in a designed focal plane. The mode-locked pulsed laser source generates a train of pulses centered at wavelength $\lambda_{o}=720 \mathrm{~nm}$ and featuring a temporal width of about $2.5 \mathrm{ps}$. The required spectral separation of the lines in the comb can be 
accomplished by directing the laser beam onto the metasurface at an off-normal angle of incidence $\theta_{i}$. In our case this angle was chosen as $\theta_{i}=45^{\circ}$. When the phase-shift induced by the metasurface mimics a diffraction grating $\left(\emptyset_{m 1}=-k_{o} \sin \theta_{i} x\right)$, the different spectral components will be decomposed and transmitted into slightly different directions around the sample normal (more details in supplementary text S3). The required focussing could be accomplished with a second metasurface acting as a cylindrical convex lens with a focal distance $\left(f_{c}\right)$ and a phase profile $\left(\emptyset_{m 2}=-k_{o}\left(f_{c}-\sqrt{f_{c}^{2}+x^{2}}\right)\right)$. However, one of the key benefits of using a phase-gradient metasurfaces is that these two optical functions can be combined in a single metasurface by engineering a phase profile $\left(\emptyset_{m}=\emptyset_{m 1}+\emptyset_{m 2}\right)$. Accordingly, the entire beam steering system is composed of only a frequency-comb source and a single metasurface.

The designed focal length is $f_{c}=1 \mathrm{~cm}$, resulting in a frequency-gradient $\frac{\Delta \omega}{d}=\frac{\omega_{o}}{f_{c} \sin \theta_{i}}=$ $2 \pi \times 5.89 \mathrm{GHz} / \mu \mathrm{m}$ (See details in supplementary text S3). Decreasing/incresing the designed focal length would result in a larger/smaller frequency-gradient and a beam steering rate. A Si metasurface based on the geometric phase $(28-30)$ is used to create the virtual frequency-gradient metasurface (Figs. 2B, C). The Si nanobeams in this device are fabricated on a sapphire substrate by electron-beam lithography and reactive-ion etching. They are $500 \mathrm{~nm}$ high, feature a rectangular cross section of $180 \mathrm{~nm} \times 110 \mathrm{~nm}$ and are spaced by $350 \mathrm{~nm}$. The entire metasurface is large (measures $8 \mathrm{~mm} \times 4 \mathrm{~mm}$ ), in order to increase the NA required for strong focusing and a larger steering angle. More fabrication details on the design and fabrication can be found in the methods section. The geometric phase is obtained using an array of Si nanoantennas with space-variant orientations (28-30) (Fig. 2B, C). The geometric phase emerging from a surface patterned with such antennas is given by $\emptyset_{m}=2 \sigma \psi(x, y)$, where $\psi(x, y)$ is the in-plane orientation of the nanoantennas and $\sigma$ quantifies the spin angular momentum for the incident light, i.e., right ( $\sigma=$ 
1) or left $(\sigma=-1)$ circular polarization. Importantly, this phase can be designed to span the full $2 \pi$ range while maintaining a uniform transmission amplitude across the metasurface. These aspects of geometric phase elements can be used to achieve high diffraction efficiency optical elements for incident beams with circularly-polarized light (See details in supplementary text S3).

The spatiotemporal intereference from the array of virtual sources creates a small-diameter steering beam. A microscope objective is then utilized to map the time-varying steering angle $\theta$ to an axial dimension $y_{1}$ at the back focal plane $\left(f_{\text {M.O. }}\right)$ of the microscope objective. Finally, a $4 \mathrm{f}$ imaging system is used to scale down the spatial extend of the beam $\left(y_{2}=-\frac{f_{2}}{f_{1}} y_{1}\right)$ to fit with the acceptance slit size of our streak camera used to measure the time dependence of the steering angle $\theta$. Parameters used in the experiment are: $\theta_{i}=45^{\circ}, f_{c}=1 \mathrm{~cm}, f_{M . O}=0.3 \mathrm{~cm} ., f_{1}=30 \mathrm{~cm}$, and $f_{2}=10 \mathrm{~cm}$.

The streak camera measurements showing that laser scanning over an angle of $25^{\circ}$ within a time period of around $8 \mathrm{ps}$ (Fig. 3). The temporal pulse width is $\sim 2-3 \mathrm{ps}$ (similar to that of input pulse), and the angular pulse width is $\sim 5-6^{\circ}$ which corresponds to a temporal pulse width multiplied by the angular scan speed as discussed earlier. Due to the temporal resolution of the streak camera being $\sim 2 \mathrm{ps}$, an interpolation algorithm is utilized to determine the temporal location of the pulse peak for each steering angle (white dots). The white dashed line represents a linear fit to this data, which is used to calculate the angular scan speed to be $3.45 \%$ ps. The yellow dashed line represents the time vs angle dependence according to $\mathrm{Eq}(3)$ that shows an approximately linear curve for the time vs angle relation and demonstrates the angular speed $\sim 2.4^{\circ} / \mathrm{ps}$. We attribute the small difference of the experimental slope and the theoretical prediction to the variations in the time delays aquired by the pulses passing through different vertical locations $y_{1}$ or $y_{2}$ in Fig. 2A. This can be induced by different factors, including slight variations in optical path lengths, 
deviations in dimensions of $\mathrm{Si}$ antennas across the metasurface as well as the dispersive properties of the objective lens and other lenses of our $4 \mathrm{f}$ imaging system that can cause different group delays across the lens surface (31). These delays can be on the order of picoseconds, but do not impact the observed results in a fundamental way.

The properties of the steered beam are controlled by both the metasurface and the frequency-comb source. While the angular resolution is determined the bandwidth of the frequency-comb source as discussed earlier, the beam steering angle-of-view (AOV) is controlled by the dielectric metasurface. Large AOV requires high numeric aperture of the cylinrical lensing action in order to provide narrow focal lines at the virtual frequency-gradient source. This is controlled by the footprint size and quality of the nano-structured metasurface. The dielectric metasurface is also responsible for the power efficiency, and steering speed (more details in spplementary text S3)

This work illustrates how a spatiotemporally varying metasurface can be created by combining a frequency comb source with a passive metasurface. A special case of such a surface, a virtual frequency gradient metasurface, is implemented and demonstrated experimentally. It demonstrates a conceptually new pathway for non-mechanical, ultrafast dynamic beam steering. Given the notable advances in on-chip frequency-comb sources, it is within reach to integrate the entire system on a single chip $(32,33)$. The proposed technology can also be expanded to realize other spatiotemporal patterns that e.g. allow for 2D raster scanning and axial scanning of focusing lenses. 
A

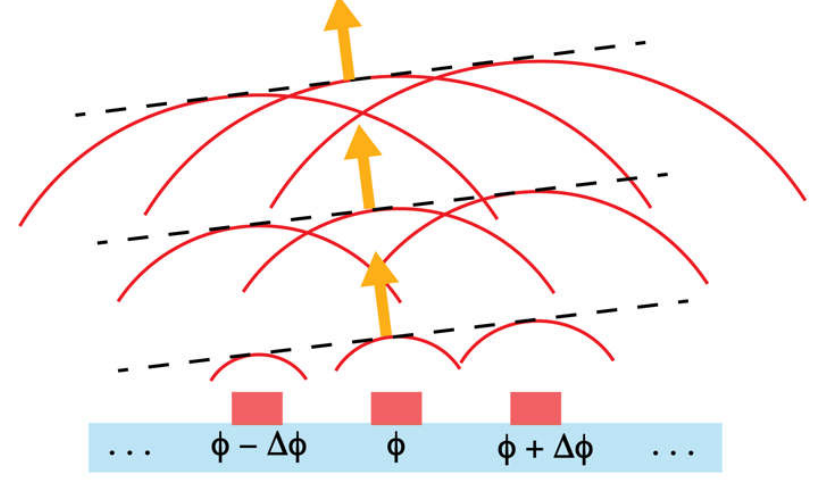

Phase-gradient Metasurface

(Static Beam Steering)

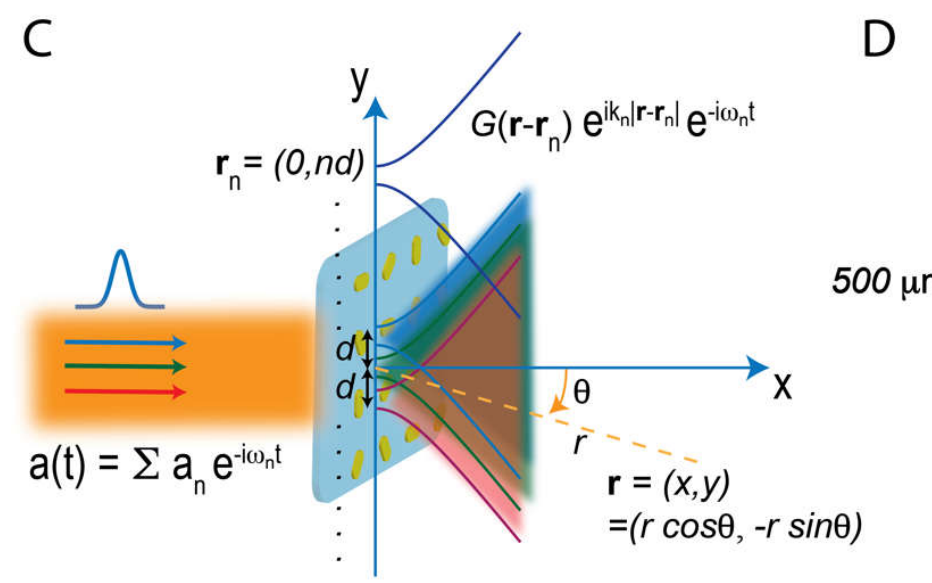

B

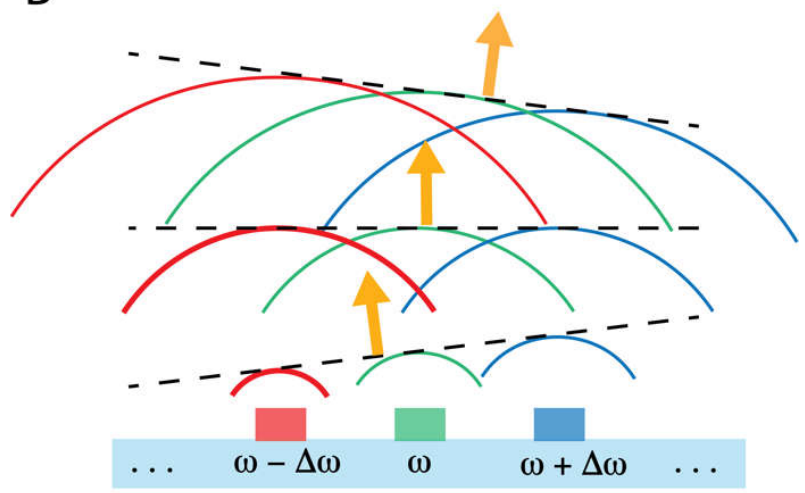

Frequency-gradient Metasurface (Dynamic Beam Steering)

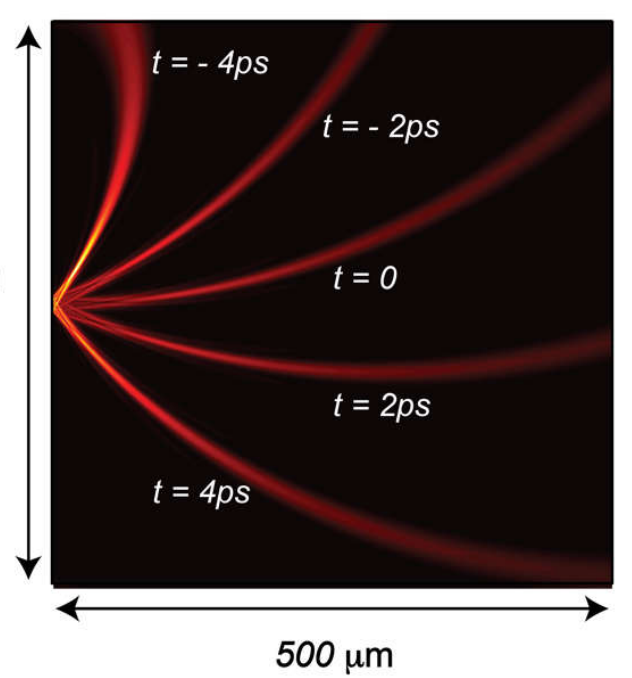

Fig 1: Theoretical demonstration of the frequency-gradient metasurface (A) Schematic of a phasegradient metasurface capable of redirecting an incident plane wave towards new, fixed direction by tilting the optical phase-front. (B) Schematic of a frequency-gradient metasurface that naturally keeps reorienting optical phase-front as time progresses, facilitating continuous dynamic beam steering. (C) Illustration of the light-matter interaction between a frequency-comb source and a passive metasurface, in which the metasurface maps each spectral line of the frequency component into a spatial optical mode $\left(a_{n} \rightarrow b_{n}(\mathbf{r})\right)$, genarating a spatiotemporal optical pattern $b(\mathbf{r}, \mathbf{t})$. (D) Light generated at different time instants by our designed frequency-gradient metasurface, consisting of 41 cylindrical sources $(2 N+1=41 ; N=20)$, a separation between sources $d=$ 
$360 \mathrm{~nm}$, a center wavelength of $\lambda_{o}=720 \mathrm{~nm}$ ((i.e. $\left.\omega_{o}=2 \pi \times 416.66 \mathrm{THz}\right)$, and $\Delta \omega=2 \pi \times$ $100 \mathrm{GHz}$.
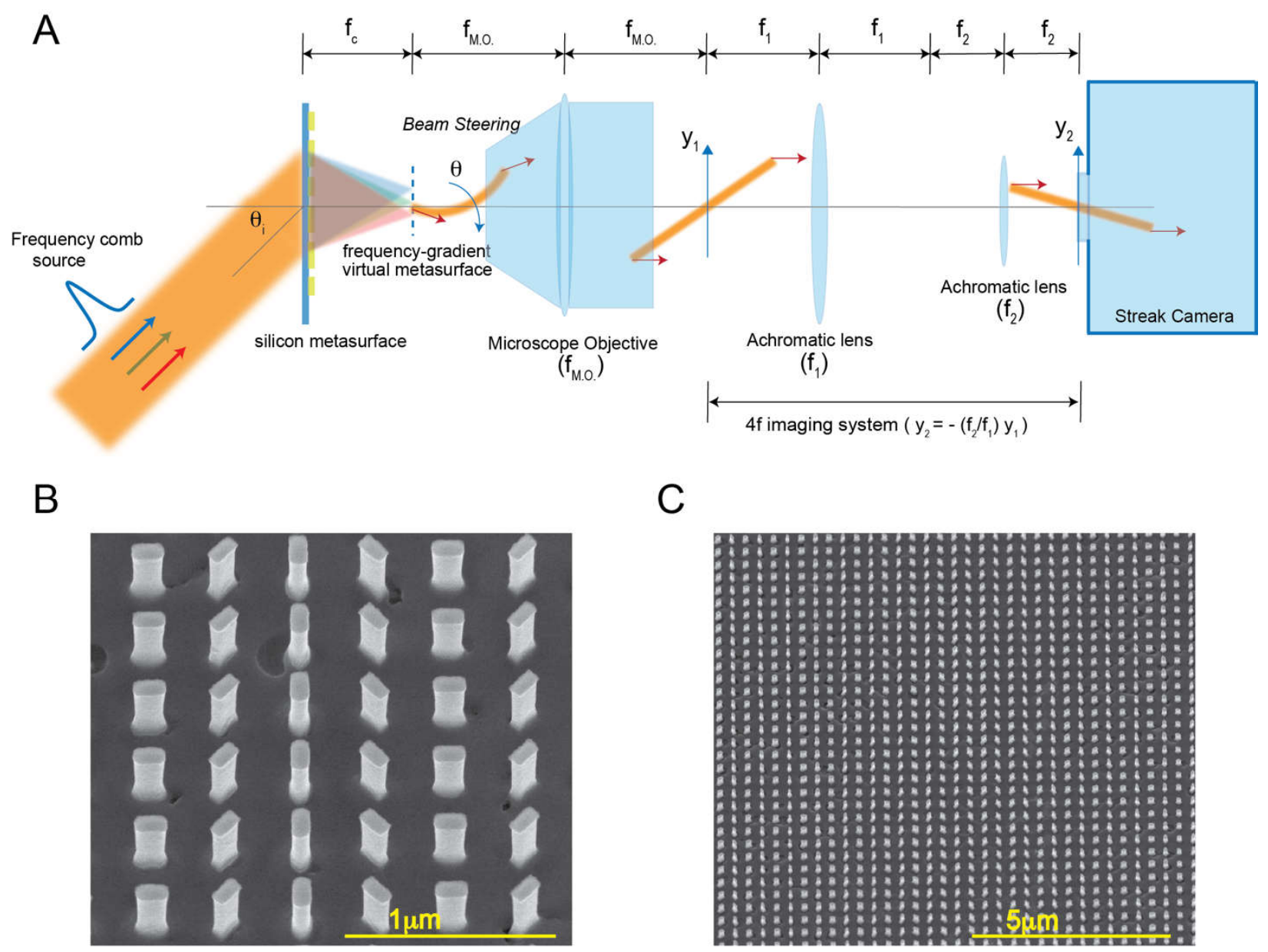

Fig 2: Experimental setup of laser beam steering using frequency-gradient metasurface (A) Experimental setup to demonstrate and quantitatively analyze the beam steering. The beam steering device consists of a pulsed laser source and a passive, phase-gradient silicon metasurface that focuses different spectral components to produce an array of focal lines that make up a virtual frequency-gradient metasurface. Measurements of steering angle $\theta$ versus time is obtained with a streak camera. Mapping of the angle $\theta$ to the streak camera is done using a microscope objective which maps to axial dimension $y_{l}$ on the back focal plane, and a $4 \mathrm{f}$ imaging system to scale down the spatial extend $\left(y_{2}=-\frac{f_{2}}{f_{1}} y_{1}\right)$ in order to fit with the slit size of the streak camera. Experimental parameters: $\theta_{i}=45^{\circ}, f_{c}=1 \mathrm{~cm}, f_{M . O .}=0.3 \mathrm{~cm} ., f_{1}=30 \mathrm{~cm}$, and $f_{2}=10 \mathrm{~cm} .(\mathrm{B}, \mathrm{C})$ 
Scanning-electron micrographs of the geometric-phase silicon based metasurface. The Si nanoantennas on top of sapphire substrate have a $180 \mathrm{~nm} \times 110 \mathrm{~nm}$ footprint, and a height of $500 \mathrm{~nm}$.

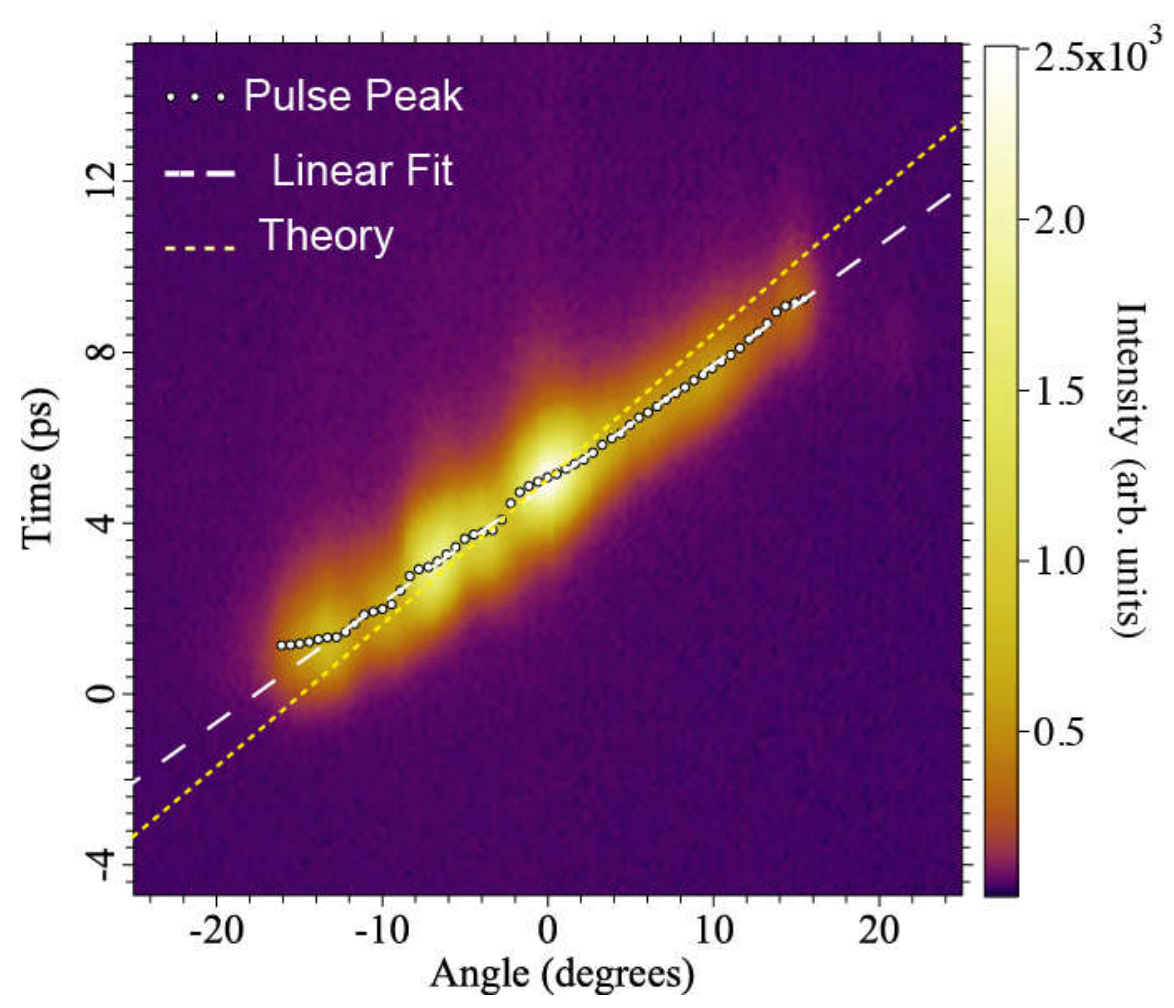

Fig 3: Experimental results of the beam steering action: Streak camera measurements after mapping each time with its corresponding beam steering angle. It demonstrates the angular redirection over $25^{\circ}$ in 8 picoseconds. The white dots denote the peak locations for the pulse at each steering angle. The white dashed line shows the linear fit used to calculate the angular speed of beam steering. The yellow dashed line shows the theoretical prediction of the time vs angle relation, assuming dispersion-less imaging optics. 


\section{References:}

1. Huang, Y. W. et al. Gate-Tunable Conducting Oxide Metasurfaces. Nano Lett. 16, 53195325 (2016).

2. Park, J., Kang, J. H., Kim, S. J., Liu, X. \& Brongersma, M. L. Dynamic reflection phase and polarization control in metasurfaces. Nano Lett. 17, 407-413 (2017).

3. Kamali, S. M., Arbabi, E., Arbabi, A., Horie, Y. \& Faraon, A. Highly tunable elastic dielectric metasurface lenses. Laser Photonics Rev. 10, 1002-1008 (2016).

4. Komar, A. et al. Electrically tunable all-dielectric optical metasurfaces based on liquid crystals. Appl. Phys. Lett. 110, (2017).

5. Gholipour, B., Zhang, J., MacDonald, K. F., Hewak, D. W. \& Zheludev, N. I. An alloptical, non-volatile, bidirectional, phase-change meta-switch. Adv. Mater. 25, 3050-3054 (2013).

6. Nicholls, L. H. et al. Ultrafast synthesis and switching of light polarization in nonlinear anisotropic metamaterials. Nat. Photonics 11, 628-633 (2017).

7. Lee, K. et al. Linear frequency conversion via sudden merging of meta-atoms in timevariant metasurfaces. Nat. Photonics 12, 765-773 (2018).

8. Zhu, Z., Evans, P. G., Haglund, R. F. \& Valentine, J. G. Dynamically Reconfigurable Metadevice Employing Nanostructured Phase-Change Materials. Nano Lett. 17, 48814885 (2017).

9. Sherrott, M. C. et al. Experimental Demonstration of $>230^{\circ}$ Phase Modulation in GateTunable Graphene-Gold Reconfigurable Mid-Infrared Metasurfaces. Nano Lett. 17, 30273034 (2017).

10. Meinzer, N., Barnes, W. L. \& Hooper, I. R. Plasmonic meta-atoms and metasurfaces. Nat. Photonics 8, 889-898 (2014).

11. Yu, N. et al. Light Propagation with Phase Discontinuities Reflection and Refraction. Science 334, 333-337 (2011).

12. Aieta, F. et al. Aberration-free ultrathin flat lenses and axicons at telecom wavelengths based on plasmonic metasurfaces. Nano Lett. 12, 4932-4936 (2012).

13. Hasman, E., Kleiner, V., Biener, G. \& Niv, A. Polarization dependent focusing lens by use of quantized Pancharatnam-Berry phase diffractive optics. Appl. Phys. Lett. 82, 328-330 (2003).

14. Lalanne, P. \& Chavel, P. Metalenses at visible wavelengths: past, present, perspectives Lalanne - 2017 - Laser \&amp; Photonics Reviews - Wiley Online Library. Laser Photon. Rev. 11, (2017).

15. Larouche, S., Tsai, Y. J., Tyler, T., Jokerst, N. M. \& Smith, D. R. Infrared metamaterial phase holograms. Nat. Mater. 11, 450-454 (2012).

16. Pors, A. \& Bozhevolnyi, S. I. Efficient and broadband quarter-wave plates by gapplasmon resonators. Opt. Express 21, 2942-2952 (2013).

17. Yu, N. et al. A broadband, background-free quarter-wave plate based on plasmonic metasurfaces. Nano Lett. 12, 6328-6333 (2012). 
18. Zhu, Z. H. et al. Metallic nanofilm half-wave plate based on magnetic plasmon resonance. Opt. Lett. 37, 698-700 (2012).

19. Papakostas, A. et al. Optical Manifestations of Planar Chirality. Phys. Rev. Lett. 90, 107404 (2003).

20. Plum, E. et al. Metamaterials: Optical activity without chirality. Phys. Rev. Lett. 102, 113902 (2009).

21. Shaltout, A., Liu, J., Kildishev, A. \& Shalaev, V. Photonic spin Hall effect in gapplasmon metasurfaces for on-chip chiroptical spectroscopy. Optica 2, 860-863 (2015).

22. Shaltout, A., Kildishev, A. \& Shalaev, V. Time-varying metasurfaces and Lorentz nonreciprocity. Opt. Mater. Express 5, 2459-2467 (2015).

23. Hadad, Y., Sounas, D. L. \& Alu, A. Space-time gradient metasurfaces. Phys. Rev. B 92, 100304 (2015).

24. Ni, X., Emani, N. K., Kildishev, A. V., Boltasseva, A. \& Shalaev, V. M. Broadband light bending with plasmonic nanoantennas. Science 335, 427 (2012).

25. Weiner, A. M. Femtosecond pulse shaping using spatial light modulators. Review of Scientific Instruments 71, 1929 (2000).

26. Weiner, A. M. Ultrafast optical pulse shaping: A tutorial review. Optics Communications 284, 3669-3692 (2011).

27. Froula, D. H. et al. Spatiotemporal control of laser intensity. Nat. Photonics 12, 262-265 (2018).

28. Bomzon, Z., Kleiner, V. \& Hasman, E. Pancharatnam-Berry phase in space-variant polarization-state manipulations with subwavelength gratings. Opt. Lett. 26, 1424-1426 (2001).

29. Bomzon, Z., Biener, G., Kleiner, V. \& Hasman, E. Space-variant Pancharatnam-Berry phase optical elements with computer-generated subwavelength gratings. Opt. Lett. 27, 1141-1143 (2002).

30. Lin, D., Fan, P., Hasman, E. \& Brongersma, M. L. Dielectric gradient metasurface optical elements. Science 345, 298-302 (2014).

31. Weiner, A. M. Ultrafast Optics. John \& Wiley Sons (2011).

32. Del'Haye, P. et al. Optical frequency comb generation from a monolithic microresonator. Nature 450, 1214-1217 (2007).

33. Ferdous, F. et al. Spectral line-by-line pulse shaping of on-chip microresonator frequency combs. Nat. Photonics 5, 770-776 (2011).

Acknowledgements: The authors would like to thank Professor Andrew Weiner for insightful discussions. Funding: This work was supported by the U.S. Air Force Office of Scientific Research (grant FA9550-14-1-0389). In addition, VMS also acknowledges partial support from by AFOSR grant FA9550-18-1-0002, and JV from the AFOSR Grant No. FA9550-17- 1-0002. J.vd G. was supported by a Rubicon Fellowship from the "Nederlandse organisatie voor Wetenschappelijk Onderzoek (NWO)”. Author Contributions: A.M.S, V.M.S and M.L.B 
developed the concept. K.L and J.V carried out the streak camera measurements. J.v.d.G and S.K fabricated the silicon metasurface. All the authors contributed to and approved the manuscript. Competing interests: The authors declare no competing interests. Data and Materials availability: All data is available in the main text or the supplementary materials. 